\title{
Mit der 2. Säule (= BVG) massiv Steuern sparen!
}

\section{J. Schmid}

Korrepondenz:

Jürg Schmid, lic. iur HSG

Geschäftsstelle FMH Services

Treuhand Speicher/St. Gallen

c/o Schmid \& Partner Treuhand AG

Zaun 55

CH-9042 Speicher

Tel. 0713442175

Fax 0713443980

E-Mail:

juerg.schmid@fmhtreuhand.ch
Grundsätzlich haben Selbständigerwerbende die Möglichkeit, 20 \% des Erwerbseinkommens oder maximal Fr. 30384 .- für die Säule 3a einzuzahlen. $\mathrm{Zu}$ beachten ist aber, dass diese Lösung für gutverdienende Selbständigerwerbende nicht optimal ist, da z. B. keine nachträglichen Einkäufe möglich sind, auf dem ganzen Reingewinn immer auch die vollen AHV-Beiträge (max. 9,5\%) zu zahlen und Einkommen über Fr. 150 000.- nicht mehr versichert sind.

Aus diesem Grunde ist die BVG-Lösung (=2. Säule) vor allem für gutverdienende (über Fr. 150 000.-) Selbständigerwerbende steuerlich weit interessanter als die Säule-3a-Lösung.

Interessant ist die Lösung vor allem darum, weil praktisch der ganze Lohn versichert werden kann, was weitaus höhere Abzüge als bei der Säule 3a ermöglicht. Da darüber hinaus die Hälfte der Prämie auch noch als Geschäftsaufwand der Praxisrechnung belastet werden kann, werden zugleich noch AHV-Beiträge gespart. Nebst diesen steuerlichen Aspekten sind auch die Risiken bezüglich Alter, Tod und Invalidität in der 2. Säule weit besser abdeckbar.

Durch einen Wechsel von der grossen Säule 3 a zur 2. Säule wird zusätzlich auch noch ein erhebliches Einkaufspotential geschaffen, können doch vor allem bei Ärzten sehr hohe, freiwillige Einkaufssummen zur Leistungsverbesserung erfolgen, welche vollumfänglich steuerlich abzugsfähig und damit sehr interessant sind.

Zusätzlich zur 2. Säule können aber trotzdem auch noch Beiträge in ein kleines Säule-3a-Konto erfolgen, nämlich maximal Fr. 6077.- pro Jahr.

Wer also in den kommenden Steuerperioden Steuern sparen will, sollte die Steuerplanung im Bereich berufliche Vorsorge für die nächsten Jahre umgehend in Angriff nehmen. Durch gezielte Massnahmen können beachtliche Steuerersparnisse erreicht werden!

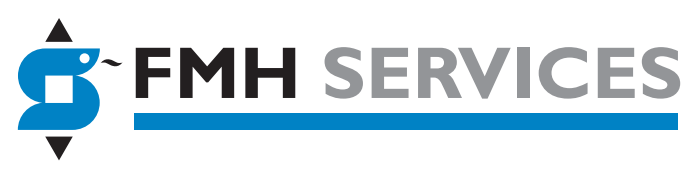

Unsere Treuhandspezialisten im deutschsprachigen Raum

Geschäftsstelle Muri b. Bern, Herr Peter Schneider, Tel. 0319518840

Geschäftsstelle Ostermundigen, Herr Harry Huwiler, Tel. 0319390139

Geschäftsstelle Olten,

Herr Peter Senn, Tel. 0622059035

Geschäftsstelle Basel,

Herr Linus Cavegn Tel. 0613195121

Geschäftsstelle Lohn-Ammannsegg SO, Herr Rolf Lehmann, Tel. 0326775442

Geschäftsstelle Muri AG,

Herr Roland Bütler, Tel. 0566640309

Geschäftsstelle Cham,

Herr Guido Schmid, Tel. 0417486290

Geschäftsstelle Sursee,

Herr Patrik Dahinden, Tel. 0419267045

Geschäftsstelle Stans,

Herr Marcel Helfenstein, Tel. 0416111821

Geschäftsstelle Zürich-Wiedikon,

Herr Christoph Lautenschlager, Tel. 014571575

Geschäftsstelle Winterthur,

Herr Urs Gross, Tel. 0522240241

Geschäftsstelle Weinfelden,

Herr Adrian Hartmann, Tel. 0716228686

Geschäftsstelle Oberuzwil SG,

Herr Martin Brenner, Tel. 0719513066

Geschäftsstelle Neuhausen a. Rheinfall, Herr Peter Oechslin, Tel. 0526755925

Geschäftsstelle Au SG,

Herr Anibal Alghisi, Tel. 0717401787

Geschäftsstelle Chur,

p. Adr. Riedi Ruffner Theus AG, Tel. 0812584646 
Ärztinnen und Ärzte, die ihre tägliche Praxis von Beginn an im Zeichen des Erfolges gestalten wollen, sollten sich schon bei der Theorie auf einen praxiserprobten, zuverlässigen und kompetenten Partner verlassen. Die FMH Services stehen Ihnen mit Rat, Tat und praktischen Dienstleistungen zur Seite.

\section{Von Theorie und Praxis}

- Beratung Praxiseröffnung/-übernahme

- Standortbeurteilungen

- Praxisplanung/-einrichtung

- Versicherungsberatung

- Seminare
- Inventar- und Goodwillschätzungen

- Treuhand

- Gemeinsamer Einkauf

- Sonderaktionen wie Kreditkarten

- Stellenvermittlung

- Inkasso

- Marketing und Kommunikation

Und vieles mehr, was Ärztinnen und Ärzte in der täglichen Praxis benötigen.

FMH Consulting Services

Burghöhe $1 \bullet$ Postfach $246 \bullet 6208$ Oberkirch • Tel. $0419250077 \bullet$ Fax $0419210586 \bullet$ mail@fmhservices.ch・www.fmhservices.ch

\section{GंFH SERVICES}

\section{Stellenplattform für Ärztinnen und Ärzte www.FMHjob.ch}

Für Fragen kontaktieren Sie Frau Logovi von der Abteilung Stellenvermittlung in Bern unter Tel. 0313591212 oder E-Mail: fmhstv@hin.ch.

\section{Service en ligne de placement et de remise de cabinets}

\section{www.FMHjob.ch}

Si vous souhaitez obtenir de plus amples informations, n'hésitez pas à prendre contact avec Madame Logovi de l'Office de placement à Berne au numéro de téléphone 0313591212 ou e-mail: fmhstv@hin.ch. 